\title{
Construction of SSI Framework Based on MVC Software Design Model
}

\author{
Yongchang $\mathrm{Ren}^{\mathrm{a}}$, Yongzhe $\mathrm{Ma}^{\mathrm{b}}$
}

\author{
School of Information Science and Technology, Bohai University, Jinzhou, P.R. China \\ a1213552916@qq.com, b1125444268@qq.com
}

Keywords: MVC; SSI framework; struts framework; spring framework; ibatis framework

\begin{abstract}
Good development framework can improve quality of software development. This paper makes research based on the MVC software design model, after thorough analysis of the current mainstream of three technical framework, combining with their characteristics and advantages to construct SSI fusion framework which is based on three layer architecture. The framework takes the struts framework as the basis, which not only integrates the struts framework and the spring framework, but also the spring framework and ibatis framework that applied respectively to the presentation layer, business logic layer and data persistence layer. All layers work collaboratively and the coupling degree is low. The SSI framework constructed in this paper is benefit for the expansion and maintenance of system in the late, which can provide guarantee for improving software development efficiency and enhancing code robustness, it plays a certain role in promoting web application development.
\end{abstract}

\section{Introduction}

With the rapid development of Internet, the software application systems which use C/S mode have gradually fade out sight of people, the application software systems based on network are being pursued. System generally is composed of three parts, the presentation layer, business logic layer and data persistence layer, in the design process, often because of their low coupling degree, which leads to complicated development process, difficult system maintenance and version upgrade, poor scalability, how to improve the coupling degree of three layers is a key. J2EE has many open source framework, such as Struts, JSF, Echo, Spring, Hibernate, JPA, iBatis and so on, which has its own unique advantages [1]. Tang Yongrui uses the SSI framework based on MVC mode to develop a set of emergency affairs management system. Zhen GuangChou makes research based on the three SSI framework technology, integrately designs and implements rural information management system [2,3]. However, the current studies have the problems of poor system reusability and low integration and difficult extension. Reference to previous studies, this paper uses the MVC software design pattern and makes full use of the advantages of Struts, Spring, Ibatis, integrating them to build SSI development framework. Making software development based on the framework can effectively solve the above problems.

\section{MVC Software Design Model}

MVC is the abbreviation of model, view and controller, namely the model-view-control, it is a kind of software design patterns which is widely used and very popular in software development. According to the level layout of model, view, and control separating an application process, collaborative work between three levels, giving full play of their respective characteristics to complete the corresponding functions, clear division of responsibilities, no influences between each other, achieving the purpose of reducing the coupling degree between layer and layer, which can largely enhance software performance, improve the system security performance, lay the foundation for later maintainable and extensible of software development. The relationship between the layers and the functions implemented are as shown in Fig. 1 [4]. 


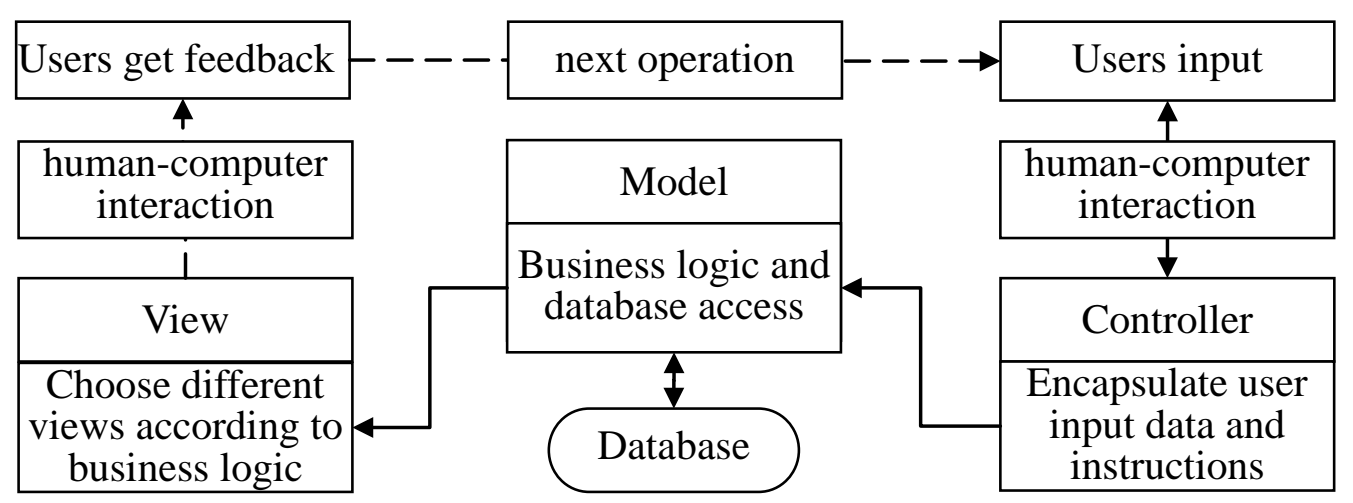

Fig.1. MVC software design pattern

Model: Its functions are to realize the most, but also the most core part, containing the business logic of the application, through collecting business data judging the business logic and database access. Multiple views can receive provided data from a model at the same time, when the business data states and logic change in the model, it will promptly inform the view layer, open service interface to facilitate the view layer access; a model can also be repeatedly used by multiple views, its code reuse can be improved. In addition, the model can be further subdivided into the data access layer, business logic layer and so on.

View: It is the user interface we often say, users operate through the view to realize interaction with system. The view layer can receive user input data, also can obtain the data of the model layer, according to different business logic select different views to display to users, at the same time provide users with a variety of data validation function [5]. In the process, the view is unable to operate the data in the model, also has no any abilities to actually handle the business, it is just as the way that output data and allows users to manipulate [6].

Controller: responsible for receiving user request, encapsulating the user input commands and data and passing to the business model. As launching points of business process, the controller plays a crucial role. It is mainly controls the direction of user data and instructions, and does not have the function of processing data, according to user's request passes to the corresponding model to handle, decides which view shows.

\section{Struts Framework}

Struts is an open source framework which adopts the MVC pattern, it is composed of three parts of the business model layer, the view layer, the business layer. The control layer is the core, which is mainly responsible for the business logic and the processing of the action and through FilterDispatcher loads implementation class. The Struts framework uses the request-response process to develop applications, the business model layer receives the called URL when users operate and encapsulates the data users input form; the control layer according to the request users send to execute the corresponding action which in the struts.xml configuration file; the view layer by matching the URL in struts.xml configuration file searches and finds returned jsp pages, the request data are returned and presented to users parsed through the browser. The framework is widely used in the web system development, because of its clear level and convenient development mode, system development efficiency is improved significantly, the workload is greatly reduced.

\section{Spring Framework}

As a lightweight open source framework, spring framework takes inversion of control and aspect oriented programming as the core, which adopts the way of declaring object to manage things, and provides the integrated framework to solve the problem of JavaEE. It is made up of seven big modules, low coupling between modules, through the presentation layer, business logic layer and data persistence layer. Through the configuration file to set up the dependent relationships between 
business objects, implementing the business logic interface access; using aspect oriented programming to cope with the difficulties that function is hardly realized by using traditional object-oriented programming, which can provide a one-stop solution for JavaEE. In the process of project development, there is a choice to deploy appropriate module according to the actual situation. In addition, spring also provides the interface combined with other frameworks, which can be perfectly combined with common framework technology such as ibatis, struts, hibernate and so on, fully play the advantages of each layer to improve system robustness and portability.

\section{Ibatis Framework}

Ibatis is a semi-automatic data persistence layer framework, including SQLMaps and DAO two types of components, SQL Maps components are used to implement the CRUD operation on relational database for Java object [7], which can access database through the DAO components. Compared with Hibernate, JPA, the characteristic of ibatis is semi-automatic. As far as hibernate is concerned, it has completely encapsulated the structure of the database, which has a set of mapping mechanism from POJO to database table; in the development process it only needs to define its mapping relationship to realize the database access, and even do not need to grasp SQL syntax. This approach is faster, but in certain circumstances, it is helpless when some complex data manipulations are needed. Ibatis well solves the problem, it allows developers to customize SQL to configure the Bean attribute values, also allows to use database to extend the standard SQL syntax [8]. Through configuring the XML file name and ID to interact with the database real-time, convenient and flexible, provides software developers with larger free space. It is widely used in small application softwares, large and medium-sized enterprise application systems, which is one of the convenient and flexible persistence frameworks at present.

\section{SSI Fusion Framework}

Although the functions of struts, spring and ibatis framework is strong, but its advantages are only limited to develop a layer of system structure, which can't complete the actual development tasks. This article uses the MVC design pattern and takes struts framework as the basis architecture to construct in the presentation layer, receive the requests and input data sent by users, can quickly through the struts XML configuration files to find the corresponding action and use technologies such as JSP to write dynamic pages to interact with users [9]; The integration of the struts framework and spring framework is constructed on the business logic layer, mapping service class interfaces in the action, drawing related interface implementation method; The integration of the spring framework and ibatis framework is constructed on data persistence layer, in the access database configuration file completing database operations in interface implementation methods of database operations, which play fully respective advantages. The framework structure is shown in Fig. 2. Specific fusion method is as follows:

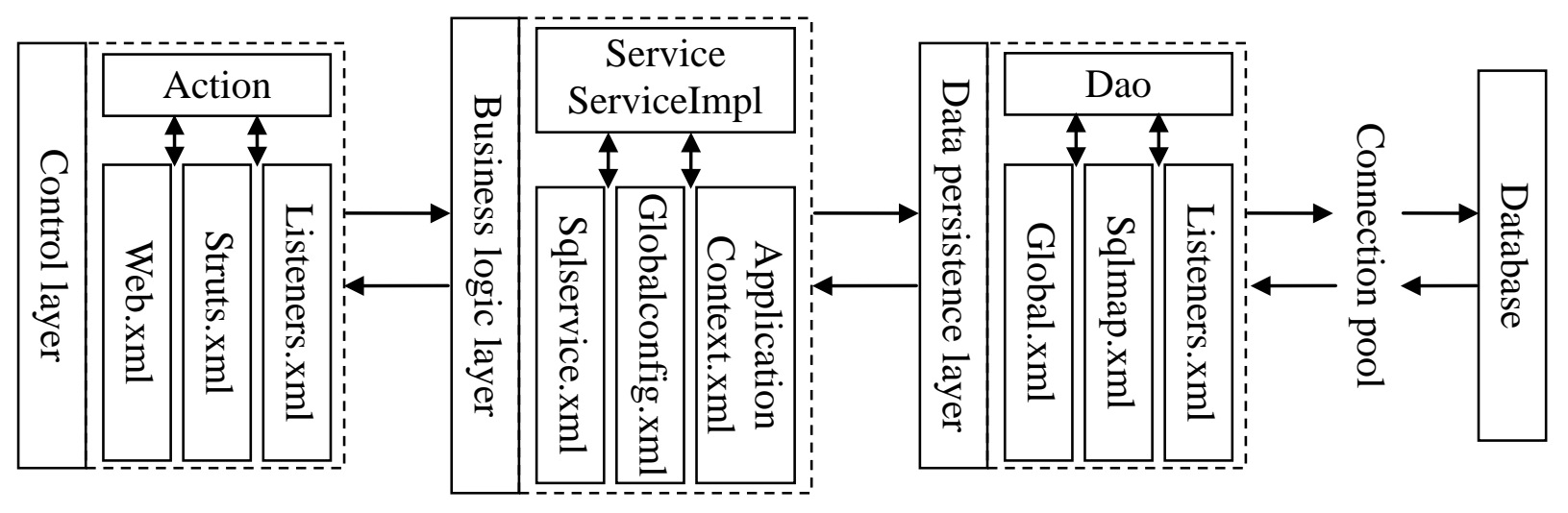

Fig.2. SSI fusion framework structure 
Struts and spring fusion: Struts framework uses the MVC pattern, which pays attention to separate the view layer and control layer, but not involves the optimization design on the model layer [10]. Spring framework is a good solution to solve the problem, which uses inversion of control mechanism to optimize the model layer, and reduces the coupling degree between the layers. Specific operation procedures are: according to the URL and operation interface which users invoke, drawing action in the struts.xml configuration file, returning the data after performing related operations, which are presented to the user in the form of JSP pages.

Code configuration is as follows:

$<$ action name="baseActionForm" path="/debugPartBack"

scope $=$ "request" parameter $=$ "reqCode"

type ="com.nuctech.sctc.ation.DebugPartBackAction">

$<$ action $>$

$<$ forward name="debugPartBack" path="/sctc/partBack/debugPartBack.jsp"/>

In order to prevent data leakage and ensure security, specifically making the user role authorization control. Dividing access permissions according to the role authorization level, the higher the level is, the more functional modules will be access; a character can represent more than one user, the data between users cannot access with each other, which realizes the integration of safe, seamless docking and loose coupling between struts and spring framework.

Spring and ibatis fusion: Spring framework mainly completes the processing of business logic and data, DAO in ibatis framework defines the interface and implementation method of accessing database and configures them in the service.xml file, ibatis carries out SQL statements operations by DAO database access. Therefore, integrating spring and ibatis framework can replace the original invocation mechanism; when dealing with business logic and data, there is no need to pay attention to the underlying implementation details, as long as find the interface associated with its implementation methods, through implementing the methods executing SQL statements, obtaining relevant data in the database, which can execute user requests quickly, improve the system response time and overall performance. When the user operation changes, there is only need to modify the underlying operating which reduces the amount of code changes, favorable for system maintenance in the late.

Code configuration is as follows:

<bean id="debugPartBackService" parent="baseTransactionProxyG4">

$<$ property name= "target" $>$

$<$ bean class="com.nuctech.sctc.manager.impl.DebugPartBackServiceImpl">

$<$ property name = "g4Dao" ref="g4Dao" />

$<$ property name ="g4Reader" ref="g4Reader" />

$</$ bean $>$

$</$ property $>$

$</$ bean $>$

Ibatis data persistence: Data persistence layer is a very important part of the model, mainly including data access service which is responsible for the communication between the database management system [11]. Ibatis realizes basic operations on the data in the XML configuration files, and mainly adopts ways of SQL to realize data interaction, which belongs to a kind of data mapping. Though ID executing SQL statements, accessing database to transfer data; during the period, the mapping layer is independent between spring and ibatis framework which is not affected.

Code configuration and implementation are as follows:

<sqlMap resource="sqlmap/nuctech/sctc/debugPartBack.ibatis.xml"/>

$<$ insert id="insertPartBack" parameterClass ="dto" >

Insert into nuc_debug_back_apply

(debug_back_id, debug_back_num, link_con_id, user_id, transactor, delivery_time, note, status, attach, create_time, values update_time, approval_user_id,isdel) 
(\#debug_back_id\#,\#debug_back_num\#,\#link_con_id\#,\#user_id\#, \#transactor\#,\#delivery_time\#,\#note\#,\#status\#, \#BOMattachHidden\#, now(),now( , \#approval_user_id\#, 0) $</$ insert $>$

\section{Conclusion}

There are many factors that affect software development, especially the software development efficiency, robustness, expansion and maintenance of system in the late. This paper integrates characteristics and advantages of the struts framework, spring framework and ibatis framework, using the MVC software design pattern to construct SSI framework, which aims to reduce the effects of key factors, and to improve the quality of software development. The struts framework is the basis architecture which plays a role in the presentation layer; The integration of the struts framework and the spring framework which applies to the business logic layer; The integration of the spring framework and ibatis framework which applies to the data persistence layer. In addition, the framework has some shortcomings, that is the way of implementing fusion of the framework and database access is mainly the configuration of the XML files, which will bring certain difficulties to the file management.

\section{Acknowledgement}

This work is supported by social science fund project of Liaoning province (L13DTQ01).

\section{References}

[1] Jiang J Y, Liu T, Liu Y. The Construction of e-business portal based on Struts, Spring and Hibernate. Beijing Municipal Science and Technology Commission, 2009, 9(9): 78.

[2] Y. R. Tang, D. M. Zhang, "Design and Implementation of Emergency Management System Based on SSI," Computer Technology and Development, vol. 24, no. 4, pp. 151-154, 2014.

[3] G. C. Zhen, W. W. Yang, "Design and Implementation of Rural Information Management System Based on SSi Framework," Computer and Modernization, vol. 215, no. 7, pp. 156-159, 2013.

[4] F. Lan, "Design and Implementation of Web Part Time Publishing and Interactive Platform," Master's degree of Electronic Science and technology University, 2012.

[5] S. Li, "Design and Implementation of Enterprise Information Management System Based on SSI2," Master's degree of Jilin University, 2014.

[6] X. Q. Wang, "Design and Implementation of Maritime Information Service Platform Based on SSi2," Master's degree of Jiangsu University of Science and Technology, 2013.

[7] K. S. Wang, "Design and Implementation of University School Training System Based on SSI," Master's degree of Suzhou University, 2013.

[8] G. Ren, "IBATIS Framework Source Code Analysis," Beijing: Publishing House of Electronics Industry, 2010.

[9] L. J. Deng, Y. F. Zhang, J. M. Li, D. X. Dong, "SSI Integration Framework Technology and Application," Journal of Zhengzhou University of Light Industry: Natural Science Edition, vol. 30, no. 1, pp. 46-49, 2015.

[10] Z. Y. Dong, "Resource Management System Slot Port Add Scheme Base on Struts Ajax Check," Journal of Zhengzhou University of Light Industry: Natural Science Edition, vol. 28, no. 4, pp. 92-96, 2013.

[11] J. Meng, "Spring-Java Lightweight Architecture Development Practice," Beijing: The People's Posts and Telecommunications Press, 2006. 